\title{
L’interculturel en environnement : Rencontre de la justice sociale et de la justice environnementale
}

\author{
Gina Thésée, Université du Québec à Montréal \\ Paul R. Carr, Youngstown State University
}

\begin{abstract}
Résumé
Le sur-développement des pays du Nord fait planer sur le monde entier des menaces sans précédent. Les changements climatiques sont les signes avant-coureurs, à long, moyen ou court terme, de ces menaces. Aux prises directement avec les premiers impacts, les populations du Sud voient leurs multiples vulnérabilités s'accentuer. Si l'approche écologiste, après des décennies de combat, a finalement reçu l'attention des mondes politique et économique à propos des défis environnementaux, ces derniers ont adroitement opéré un virage conceptuel significatif: l'environnement et l'éducation relative à l'environnement (ERE) ont cédé la place au développement durable et à l'éducation au développement durable (EDD). Or, dans cette refonte conceptuelle, le modèle hégémonique du développement à l'occidental n'est pas remis en question. Il poursuit, entre autres, ses visées impérialistes en muselant toujours les cultures qu'il domine, tout en invalidant les savoirs dont elles sont porteuses. Il y a tout lieu de parler ici d'un racisme épistémologique. Ce texte propose quelques pistes de réflexion pour une rencontre des cultures en environnement où la justice sociale et la justice environnementale se conjuguent.
\end{abstract}

\begin{abstract}
Unprecedented threats hover over the entire world as a result of overdevelopment of Northern countries. Climate changes are the long-, medium-, and short-term forerunners of these threats. In direct grip of the first impact of the problems, the people of the South witness an increase of their multiple vulnerabilities. After decades of struggles, if the ecological approach has finally received the attention of the political and economical world authorities regarding the environmental challenges, these latter have skilfully created a significant conceptual change of policy: the environment and education related to environment (ERE) have been replaced by sustainable development and education for sustainable development (ESD). However, in this conceptual remake, the hegemony of the Occidental development model was not challenged. So this model pursues, among other things, its imperialist designs by always silencing other cultures under its domination, while invalidating the knowledges embedded in these cultures. One can speak of an epistemological racism. This paper proposes some means of reflexion to create a meeting place between cultures on the environment where social justice and environmental justice can be of the same mind.
\end{abstract}


Plus il croise de ses semblables, plus l'Homme devient humain ». Le respect et la reconnaissance des savoirs sont un préalable incontournable à la rencontre des autres et à ce formidable enrichissement que permet la diversité des cultures. (Yamgnane, 1999, p.8)

\section{Le problème : le sur-développement}

Aux expressions " pays sous-développés » et " pays en voie de développement », il y a lieu désormais d'opposer celles, non moins problématiques et combien plus menaçantes, de "pays sur-développés " et de "pays en voie de surdéveloppement ». Tandis que les premières concernent principalement le Sud, les secondes concernent principalement le Nord et sa suite, c'est-à-dire, les pays dits émergents comme le Brésil, la Chine et l'Inde. Cependant, les menaces climatiques générées par les activités industrielles et les modes de vie du Nord affectent directement les populations du Sud faisant planer sur elles le spectre de leurs multiples vulnérabilités. Il faut noter cependant que la menace climatique n’est ni environnementale, ni économique, ni politique ou éducative. Elle englobe ces quatre dimensions et plus; elle révèle une crise civilisationnelle incluant une crise du savoir. La destruction de l'environnement montre avec force que la rationalité économique sur laquelle s'est construite la modernité occidentale a atteint son point limite (Leff, 2006, p.185).

Un virage conceptuel significatif : de l'environnement au développement durable Bien que l'environnement soit un sujet préoccupant depuis le début des années soixante, avec la parution en 1962 du «Printemps silencieux » de la biologiste naturaliste Rachel Carson, ce n'est que récemment que les enjeux environnementaux ont officiellement pénétré les agendas politiques et économiques. L'influence de la fondatrice du mouvement écologique moderne, qui attirait l'attention sur le biocide causé par l'industrie chimique, aura mis une quarantaine d'années pour opérer une prise de conscience dans les sphères des pouvoirs politiques et économiques. Et voici qu'aussitôt intégrés, les concepts écologiques : «environnement » et «éducation relative à l'environnement (ERE) » sont effacés au profit des concepts économiques : "développement durable » et " éducation au développement durable (EDD) ». Les dérapages du concept de développement durable se font déjà sentir dans les discours sur les enjeux environnementaux (Sauvé, 1996). Les échanges de crédits du carbone en sont un bel exemple. Cette habile formule a été élaborée pour permettre aux pays producteurs de gaz à effet de serre (GES) de continuer à polluer tout en se donnant bonne conscience en y associant les pays économiquement pauvres. Le commerce du carbone ne fait que renforcer la position des différents acteurs du 
domaine des énergies fossiles tout en provoquant « un nombre croissant de conflits militaires et en aggravant les injustices sociales et environnementales ».

Le marché du carbone crée des droits transmissibles de déverser dans l'air, les océans, le sol et la végétation, des volumes de carbone qui dépassent de loin la capacité d'absorption de ces systèmes. Ces droits, équivalant à des milliards de dollars, seront octroyés à titre gracieux aux plus grands émetteurs de gaz à effet de serre des secteurs de l'électricité, du fer, de l'acier, du ciment, du papier et à d'autres encore dans les nations industrialisées, qui ont déjà provoqué la crise climatique et qui exploitent déjà au maximum les systèmes mentionnés. En revanche, le coût de la réduction future de l'utilisation des combustibles fossiles retombera probablement de manière disproportionnée sur le secteur public, les communautés, les peuples indigènes et les contribuables (Déclaration de Durban de 2004 sur le commerce du carbone, 2006, p. 200)

$\mathrm{Au}$ Québec et ailleurs au Canada, les dénominations successives du ministère responsable le démontrent : le Ministère de l'environnement est devenu le Ministère du développement durable et a à sa tête, est-ce étonnant, l'ex-ministre de l'industrie et du commerce. Quel virage conceptuel! Ou plutôt, quelle adroite récupération! La rationalité occidentale globalisée continue de dicter les orientations en matière environnementale sous couvert d'un discours sur le développement durable qui ne remet pas en question la logique économique désastreuse sur le plan écologique (Leff, 2006, p.185). Le développement peut-il être à la fois la cause du mal et le remède à ce même mal? Devrait-on le considérer comme un mal nécessaire? Quelles sont les implications de ce modèle économique pour la condition humaine et pour la justice sociale? Et enfin, quels sont les enjeux pour l'éducation?

\section{L'éducation relative à l'environnement et l'éducation aux sciences}

«L’éducation relative à l'environnement n’a pas commencé en 1987 avec le rapport Brundtland, elle est pratiquée de tout temps dans les sociétés traditionnelles.... Il serait urgent d'aller puiser dans leurs démarches... » (Pardo, 2002, p.1)

L'éducation relative à l'environnement (ERE) propose une perspective sociocritique des enjeux environnementaux. Elle se préoccupe de la construction du réseau des relations et s'intéresse à des questions relatives au développement des personnes et des groupes sociaux en relation avec leur milieu de vie (Sauvé, 1997). Elle revalorise l'expérience des personnes et des communautés tout en leur redonnant le sceptre de la parole. Elle établit des liens horizontaux qui rompent la 
hiérarchie traditionnelle des savoirs et des pouvoirs. Fondée sur la posture critique de l'École de Vienne (Habermas, Adorno) et sur la pédagogie de Paulo Freire (2005), elle vise la prise de pouvoir (empowerment) des acteurs des milieux concernés via la conscientisation et l'action solidaire. Par contre, si l'ERE offre un cadre de départ intéressant pour la formulation des problématiques environnementales des groupes dominés, marginalisés ou exclus, elle demeure trop discrète, pour ne pas dire muette, en ce qui a trait aux spécificités des relations de pouvoir inéquitable qui leur sont liées, par exemple le racisme, le sexisme, le classisme. Il nous semble aussi qu'elle fait l'impasse sur le rapport inéquitable des savoirs inhérent au rapport inéquitable des pouvoirs. Ne pas reconnaître l'effet insidieux et dévastateur du rapport inéquitable des savoirs, c’est esquiver une bonne part des problématiques éducatives (McLaren, 2007).

Cela étant dit, il parait aujourd'hui aberrant d'avoir confié l'éducation relative à l'environnement aux seuls enseignants de sciences dans les programmes d'éducation aux sciences tels qu'ils ont été élaborés jusque dans les années quatre-vingt-dix (Thésée, 2006). Non seulement les visées de ces programmes divergent profondément de l'ERE, mais plus inquiétant encore, la représentation positiviste des sciences et l'approche pédagogique culturaliste encore privilégiées dans l'enseignement des sciences naturelles est en flagrante contradiction avec les principes fondamentaux de l'éducation relative à l'environnement.

\section{Le modèle de développement à l’occidentale}

Le développement tel que compris jusqu'à présent a conduit à des logiques politiques, économiques, sociales, environnementales et éducatives où l'hégémonie culturelle occidentale a rencontré peu de résistance. Ce modèle universel fournit le cadre dans lequel les moyens, les stratégies, les objectifs, les buts et la finalité même de l'existence sociale sont définis et mis en œuvre : il s'agit de la chosification de la nature, de sa mise en marché et de sa consommation. En corollaire, les dynamiques sociales sous-jacentes se révèlent la chosification de l'Autre dans un processus d'invalidation, de marginalisation et d'exclusion. Bien que ces dynamiques concernent derechef des personnes et des groupes sociaux, il faut aussi tenir compte des savoirs invalidés, marginalisés et exclus dont ces personnes et groupes sociaux sont porteurs. Au mieux, les exclus sont tolérés sans leurs savoirs déclarés "non-savoirs »; au pire, ils se voient invalidés ainsi que leurs savoirs. Dans l'intermédiaire, des savoirs invalidés auront pu être récupérés mais sans aucune reconnaissance de leur origine légitime. En fait, comprendre l'environnement sans problématiser les relations inéquitables des pouvoirs et des savoirs parait aujourd'hui inconcevable. La prise de conscience des impacts en matière de changements climatiques accentue les impasses du modèle de développement dominant du Nord à l'œuvre aussi bien au Nord que dans les pays du Sud (Sharma, 2006, p.139). 
Le racisme scientifique

Certains auteurs laissent entendre que le racisme est une doctrine politique dont les défenseurs, à l'occasion, recourent aux résultats de la "science » pour confirmer l'existence de "races inférieures » (Thuillier, 1988). Or, la théorie " scientifique » raciale élaborée par les biologistes et les généticiens des XIXe et XXe siècles a fourni une catégorisation clé permettant de comprendre les pressants problèmes de l'époque, entre autres : l'avenir des Amériques, le succès des Européens dans les tropiques, l'extinction des peuples, le rôle de la GrandeBretagne en Europe (Stepan, 1982, citée par Willinsky, 1998, p.163). De plus, l'abolition de l'esclavage dans l'empire britannique en 1833 a suscité en parallèle une frénésie chez les scientifiques qui se targuaient de disséquer les différences raciales apportant ainsi des bémols à la question des droits humains des peuples nouvellement « émancipés ».

De la catégorisation de Pline en Homo sapiens et Homo monstrous, qui inspira Linné en 1758 dans son Systema Naturae, jusqu'à la publication de The Bell Curve de Herrnstein et Murray en 1994, de nombreux écrits scientifiques viendront sceller la représentation sociale de certains groupes d'humains comme des êtres physiquement, moralement, socialement, culturellement, technologiquement et spirituellement inférieurs. Ce fut une entreprise interdisciplinaire systématique de disqualification de l'Autre. Notons-en quelques-uns :

- De l'inégalité des races humaines, de Gobineau en 1853, dans lequel l'auteur établit la supériorité de l’Européen.

- Origin of species - By Means of Natural election or the Preservation of Favored Races in the Struggle for Life, de Darwin en 1859, dans lequel l'auteur propose le cadre théorique qui permettra d'élaborer et de justifier des théories racistes de même que des programmes d'eugénisme.

- Histoire de la création, de Haeckel (sans date), dans lequel l'auteur propose un scéma où l'Indo-Européen est placé dans le rameau qui s'éloigne le plus de l'homme-singe ancestral.

- L'espèce humaine, de Quatrefages en 1890, dans lequel l'auteur souligne que «les négrophiles ont tort de dire que le Nègre tel qu'il est l'égal du Blanc ».

- Les bizarreries des races humaines, de Coupin en 1905, dans lequel l'auteur propose un récit de voyages, d'aventures et des «faits les plus intéressants, les plus curieux», puisés dans "presque exclusivement les races sauvages ou à demi-civilisés ».

- Essays on Eugenics, de Galton, en 1909, dans lequel l'auteur, cousin de Darwin et fondateur de l'eugénisme, confirme «scientifiquement» 
l'inégalité des races humaines et propose un programme eugéniste ayant pour but de contrôler la pureté des races dites supérieures.

- Heredity in Relation to Eugenics, de Davenport en 1911, dans lequel l'auteur, célèbre avocat de l'eugénisme aux Etats-Unis, dénonce les mariages inter-raciaux en soulignant leurs résultats dysharmonieux au physique comme au moral.

- Human Heredity, de Fischer, Bauer et Lenz en 1931, dans lequel les auteurs reprennent à leur compte la théorie eugéniste pour justifier l'idéologie de supériorité raciale aryenne prônée par les Nazis.

- L'agression, une histoire naturelle du mal, de Lorentz en 1940, dans lequel l'auteur, Prix Nobel de physiologie et de médecine, affirme : «Il faudrait, pour la préservation de la race, être attentif à une élimination des êtres moralement inférieurs encore plus sévère qu'elle ne l'est aujourd'hui ».

- The Bell Curve : Intelligence and Class in American Life, de Herrstein et Murray en 1994, dans lequel les auteurs de Harvard se basant sur la corrélation entre la mesure du quotient intellectuel et les catégories raciales, infèrent une interprétation politique en n’hésitant pas à demander la suppression des programmes d'équité et de justice sociale. Sur l'échelle du QI qui fait encore épisodiquement la manchette dans les média, les « races » n’occupent pas le même rang.

La hiérarchie des groupes d'humains s'est opérée d'abord selon des observables phénotypiques: couleur de la peau, dimensions du crâne, texture des cheveux, forme du nez, taille, etc. Puis, la hiérarchie s'est étendue à la culture, notamment, les langues (dénommées patois), les accents (ridiculisés petit-nègre), les us et coutumes (dénommés folklore), les degrés technologiques (péjorativement dénommés primitifs), de même que les rapports au temps (jugés comme de la lenteur), les rapports à l'espace (classifiés naturels...entendez non culturels), les rapports aux autres (jugés grégaires...entendez hordes de...), les rapports à l'environnement (invalidés en ignorance).

À propos des "Noirs ", nature et culture, soudées par un quotient intellectuel héréditaire et "mesuré déficient ", sont disqualifiées d'un seul et même geste conceptuel. Nulle échappatoire possible: ni dans l'espace (la disqualification est systématique), ni par la discrétion (la disqualification est visible), ni dans le temps (la disqualification est rémanente), ni avec les générations (la disqualification est transmise), ni avec des conversions (la disqualification est innée). Il y a lieu ici de distinguer le caractère aggravé et aggravant du racisme anti-Noirs (Dei, 1996). Les scientifiques ont voulu rendre la nature complice du crime d'inégalité politique et sociale (Stephen Jay Gould, 1981, cité par Willinsky, 1998, p.169). Le racisme est une chape de plomb qui se 
referme à plusieurs tours sur des captifs éveillés mais encore incrédules. La « faute originelle» des personnes racisées, c'est-à-dire discriminées en raison de leur appartenance raciale, était d'abord anatomique puis culturelle; elle devient peu à peu mnésique. Le devoir de mémoire n’a pas encore donné ses fruits.

Le déni comme slogan : «le racisme ne peut exister puisque les races n’existent pas »

La rémanence épistémologique est tenace et tous ces a-priori pernicieux homologués et catalogués en "savoirs », suintent dans tous les aspects du champ éducatif qu'ils soient d'ordre historique, pédagogique, didactique, éthique ou esthétique. Ici, la dynamique à l'œuvre est le déni. Déni du racisme parce que déni des races. Déni des races parce que, c'est (encore!) prouvé « scientifiquement », il n'y en a pas. La chape de plomb se referme de nouveau, cette fois, sur toute possibilité de rétorquer. La parole des exclus demeure muette, leur cri semble en voie de tétanisation.

Dépossédés des outils conceptuels qui ont mené à leur disqualification, les personnes et les groupes concernés se retrouvent piégés dans des voies sans issues : nier les races et nier le racisme; ou nier les races et reconnaître le racisme; ou reconnaître les races et nier le racisme, ou encore reconnaître les races ainsi que le racisme. Dans l'une ou l'autre posture, la position est intenable; la personne se sent en porte-à-faux. Dans le dernier cas, soit reconnaître les races et le racisme, leur position académique devient intenable tant elle suscite de suspicion, réprobation, de commentaires sulfureux ou de comportements d'éclat. Car, il n'est pas politiquement correct, encore moins scientifiquement et académiquement, d'entreprendre d'analyser un concept qui s'est élaboré durant des siècles, à l'échelle mondiale, à coups de constructions et de déconstructions théoriques, de projets de recherche et de publications scientifiques, et qui continuent à avoir des incidences néfastes majeures sur les histoires sociales de millions d'individus partout sur la planète.

Racisme épistémologique

À l'instar de Foucault (1969), nous voyons ces discours muselés comme autant de savoirs invalidés dont sont porteurs les personnes et les groupes exclus selon le critère de leur appartenance raciale. Ainsi, nous reconnaissons qu'il existe bel et bien un "racisme épistémologique » d'envergure systémique qui lamine des personnes ciblées dans leur rapport au savoir, c'est-à-dire, dans leur rapport à l'autre (le social), leur rapport au monde (l'épistémologique) et leur rapport à soi (l'identitaire). Le racisme épistémologique ne signifie pas que les chercheurs de telle allégeance sont ouvertement ou même indirectement racistes comme individus; ce concept se classe dans la catégorie de racisme civilisationnel (Scheurich et Young, 2002, p.51). Le racisme épistémologique pose que les 
épistémologies de recherche, quelles qu'elles soient, émergent du contexte historique et social du groupe racial dominant, homme blanc européen d'origine, et qu'en conséquence, ces épistémologies reflètent logiquement et renforcent l'histoire sociale de ce groupe dominant, tout en excluant les épistémologies autres telles que celles des gens de couleur en général, des Autochtones des autres continents, des femmes. (Scheurich et Young, 2002). Autrement dit, les ontologies (principes de base), les épistémologies (savoirs construits), les axiologies (valeurs qui orientent) et les méthodologies (procédures adoptées) sont inextricablement liées aux histoires sociales spécifiques des groupes sociaux qui les construisent.

Celles et ceux qui se voient exclus de la cathédrale euro-centrique de la connaissance en ressentent profondément les conséquences. Celles-ci sont multiples : la représentation problématique ou pathologique des groupes racisés; l'internalisation des conceptions de soi disqualifiantes par ces groupes; l'obligation de revêtir des savoirs prêts-à-penser tissés dans l'épistémologie dominante déclarée neutre; l'obligation d'accepter des épistémologies qui se révèlent hostiles à leur groupe d'appartenance; la nécessité d'une appartenance culturelle hybride (Scheurich et Young, 2002).

\section{Colonialisme et néo-colonialisme}

Depuis le quinzième siècle occidental, la triade omnipotente scientifico-militaroreligieuse a érigé en système la domination des territoires du monde ainsi que des peuples qui y vivent. Animée de sa volonté impérialiste, elle s'est lancée dans une entreprise de découverte et de conquête. Aujourd'hui, le colonialisme s'est revêtu de nouveaux atours, il a mué en néo-colonialisme. Au plan commercial, c'est l'OMC qui est en charge (voir les obstacles aux OMD, Khor, 2006, p.67); au plan financier, le FMI et la Banque mondiale; au plan militaire, c'est l'OTAN; au plan religieux, ce sont les encycliques du Vatican ou encore les sermons hautement médiatisés des évangélistes états-uniens; au plan culturel, les technologies, les arts virtuels, les arts visuels et la musique donnent le ton.

Par ailleurs, comme l'ont si bien démontré Fanon (1967) et d'autres, le colonialisme est un état de pensée qui ligote ensemble, colonisateurs et colonisés, dans une conscience coloniale soudée et qui semble à toute épreuve. Les contours psychologiques du colonialisme sont flous mais, néanmoins, efficaces à maintenir chacun de son côté assigné de la barrière des castes, classes ou races. Le colonialisme affecte la pensée de même que les corps et secrète des forces qui érodent les sociétés colonisées dans leur humus culturel.

Géographie différenciée des menaces et de leurs conséquences

Les menaces environnementales, qu'elles soient climatiques, chimiques, nucléaires, biologiques ou bactériologiques, reproduisent en tous points 
l'échiquier mondial des constructeurs et vendeurs d'armes, des gens d'affaire cupides et insatiables, des multinationales ennemies de l'éthique, des politiques électoralistes, du positivisme aveugle, et surtout, de la bonne conscience des bonnes gens convaincues d'être seuls détenteurs de la raison. Les conséquences à court terme quant à elles reproduisent en tous points l'échiquier mondial des vaincus, des néo-colonisés, des expatriés, des exploités, des assistés, des attaqués, des défavorisés en général qui ne font que voir croître année après année leur degré de vulnérabilité. Par contre, à moyen et à long terme, les enjeux concernent l'échiquier mondial dans sa globalité et sa totalité.

\section{Quand l'environnement seul semblait menacé}

L'environnement n'est plus la chasse gardée des écologistes et des poètes. Et, par quelle ironie a t-il pu sembler appartenir au seul champ des sciences de l'environnement dites exactes? De plus, que «l'économie » ait la même racine étymologique que «l'écologie », cela n'en fait pas pour autant un allié, loin de là. Par ailleurs, le politique, toujours à la remorque de l'économique, opère un balai où signature de traités, non respect des signatures et non signature de traités s'équivalent. Le Canada en est un bel exemple. D'un certain point de vue, l'éducation pour l'environnement, en ciblant d'abord de manière étroite le redressement des comportements domestiques, a donné lieu à la banalisation des défis environnementaux. Le sort de la petite planète bleue parait en effet dépendre de la réponse des personnes aux quatre $\mathrm{R}$ injonctifs : Réduire! Récupérer! Recycler! Réutiliser! C'était le temps où les pays pollueurs s'imaginaient avoir encore la possibilité de penser (globalement) aux solutions et de panser (localement) les blessures environnementales. Seul l'environnement semblait alors menacé. La feuille de route du Canada est d'autant plus paradoxale qu'elle présente, d'une part, des chapitres de vertu environnementale entérinée par la signature du protocole de Kyoto, suivie du non respect de cette signature et, d'autre part, des chapitres d'invalidation, de marginalisation et d'exclusion des Peuples Autochtones dont les cultures favorisaient un rapport à la nature dans l'harmonie et le respect.

Quand c'est le développement lui-même qui est menacé

Quoiqu'hésitante, la reconnaissance des changements climatiques est venue changer la donne. Ces changements observés et entérinés récemment par le GIEC (groupe international d'étude du climat) font désormais planer une menace sérieuse sur le développement lui-même de même que le progrès promu «valeur " par excellence, sur l'échelle occidentale. C'est en ce sens qu'il faut comprendre l'engagement politique et économique des uns aussi bien que le non engagement ou encore le retour sur l'engagement des autres. Chacun a à cœur son développement selon une vision très étroite de ce dernier. Ironiquement, le 
concept de mondialisation rend très bien compte des liens étroits entre la lutte face aux changements climatiques et la responsabilité de tous les acteurs en présence.

Les paradoxes du développement face aux changements climatiques

On constate de nombreux cas où le développement économique a préséance sur le développement social. Réconcilier les deux n’est jamais facile puisque la pression exercée par l'extérieur fait en sorte que les choix pour les pays émergents (ou plutôt en voie de sur-développement) sont très limités. Subordonnés aux injonctions néo-libérales, ces états voient leur souveraineté sans cesse érodée. Par exemple, pour obtenir des prêts de la communauté internationale, notamment de la Banque mondiale ou du Fonds monétaire international, les pays bénéficiaires sont tenus de faire des compromis qui se révèlent souvent désastreux pour la sauvegarde de leurs richesses naturelles autant que pour la qualité de vie de leurs citoyens.

Ainsi, le Brésil vit de nombreux paradoxes face au développement, aux changements climatiques, à la pauvreté et à l'injustice sociale. Huitième plus grand pollueur de la planète en matière d'émissions de gaz à effet de serre et troisième des pays émergents après la Chine et l'Inde, le Brésil voit diminuer chaque année la superficie de la Forêt amazonienne, une perte non seulement pour l'environnement mais aussi pour les communautés autochtones qui dépendent de la richesse de ces terres et qui sont désormais vouées à la pauvreté.

Le phénomène du sur-développement économique en Chine témoigne aussi de la disparité grandissante entre riches et pauvres. Pendant que Pékin s'apprête à accueillir les Jeux Olympiques en 2008, on reconnaît la dégradation de l'environnement en ce qui concerne la qualité de l'air et de l'eau, et la dégradation du climat social, obstacle à un développement véritablement durable. Bien que les États-Unis aient octroyé le statut préférentiel à la Chine en ce qui concerne les échanges commerciaux, il reste que ce dernier a des normes particulières, surtout en ce qui concerne les droits de la personne, les droits des travailleurs et l'environnement (Dai Jinhua, 2006).

L'Inde fournit d'autres exemples où l'engagement pour le développement et le souci pour l'environnement et la justice sociale peuvent être mis en doute. Comment concilier les disparités entre les avancées scientifiques et technologiques, d'une part, et l'indigence flagrante de millions de personnes, d'autre part? L'Inde peut impressionner dans les médias internationaux et nationaux par sa vigueur économique, il n'en reste pas moins que les populations sont toujours aux prises avec la corruption, l'endettement, le système de castes qui renforcent les inégalités, le manque d'accès aux services de base ainsi que les déplacements forcés donnant lieu à des réfugiés environnementaux (Raina, 2006). 
En effet, la lutte contre la pauvreté place les pays dits émergents dans une situation très différente de celle des pays sur-développés. La pauvreté a été utilisée par l'ONU, en 2005, comme indicateur central dans l'élaboration des objectifs du Millénaire pour le développement (OMD). Ses manifestations sont multiples, comme le démontre Focus on the Global South (2006, p.31-36):

1) Le problème de l'accès (logement, eau, éducation, soins de santé, énergie)

2) La pression sur les ressources et les processus communautaires

3) La quantité, la qualité et la stabilité de l'emploi

4) L'appauvrissement des Peuples indigènes et des minorités ethniques

5) L'érosion des mécanismes de lutte face aux vulnérabilités

6) La dislocation familiale et sociale

7) La perte de pouvoir politique et économique (disempowerment)

8) La dégradation environnementale et la pollution

9) L'écart entre les genres et les conditions de vie des femmes et des filles

10) La vulnérabilité des enfants aux conditions de pauvreté et de souséducation

11) La corruption généralisée

12) La violation continue des droits de la personne

Quelques vignettes illustrant le chevauchement de l'inéquité environnementale et de l'inéquité sociale

Les catastrophes environnementales qui ravagent la planète semblent toujours provoquer des effets plus néfastes chez les gens marginalisés, exclus des réseaux du pouvoir. McLaren (2007) souligne les processus ancrés dans un néolibéralisme qui, en privilégiant les intérêts économiques plus que la valeur sociale, isolent davantage les pauvres et ce, à la fois dans leur propre pays que dans l'économie internationale globalisée. En fait, on remarque, que de manière aiguë, il y a chevauchement des vulnérabilités environnementales et des inéquités sociales. Les quelques exemples qui suivent illustrent ce chevauchement.

\section{1) L'ouragan Katrina à la Nouvelle Orléans en 2005}

Face aux images offertes par la couverture de l'ouragan Katrina en juillet 2005, les téléspectateurs du monde entier ont assisté en direct au "spectacle " de la conjugaison de l'injustice sociale, pour ne pas dire le racisme, et de l'injustice environnementale. Malgré les informations et recommandations qui ont été fournies au Congrès américain et qui prévoyaient un désastre à la Nouvelle Orléans (Bunch, 2005), les mesures nécessaires n’ont pas été prises pour le prévenir : la question environnementale ne figurait pas dans l'équation politique. Les sinistrés, les personnes les plus vulnérables et les plus démunies, se sont 
révélés aussi, par la couverture médiatique, des personnes majoritairement de race noire (Rickford, 2005; Media Awareness Network, 2007). Avec tant d'attention et de ressources tournées vers l'Iraq, la dévastation de la Nouvelle Orléans a été présentée par les médias comme un acte de nature incontournable (Act of God). Cependant, la confusion, le paternalisme et surtout les délais de réaction des autorités responsables de la protection civile ont fait comprendre à la communauté internationale observatrice que l'ouragan Katrina avait dévoilé une situation de racisme pur : la réponse du gouvernement aurait été fort différente s'il s'était agi de victimes majoritairement de race blanche. Certains responsables ont même démontré plus de souci au sujet des installations pétrolières qu'au sujet de la vie et de la sécurité des sinistrés.

\section{2) La guerre en Irak depuis 2003}

L’impérialisme militaire et ses impacts sur l'environnement en Iraq sont évidents bien que les médias occidentaux gardent le silence sur cette dimension (Payne, 2005). « À part les dégâts causés aux infrastructures, les souffrances et les décès de civils que recouvre le terme cynique de "dégâts collatéraux ", toutes les guerres ont un impact sur l'environnement. L'étendue des conséquences pour l'environnement dépend de la durée du conflit, des armes utilisées et du type de terrain et d'écosystème où le conflit se déroule ». Cette citation est d'Arne Jerneloy (2003) qui fut un expert de l'ONU sur les catastrophes environnementales.

Causée par le passage des engins de guerre et les bombardements, la rupture de ce que les Arabes appellent la "peau du désert ", cette croûte de sable et d'argile cuits par la chaleur, provoque la libération dans l'air de fines particules de sable qui, à leur tour, ont des impacts sur l'environnement, la santé et les infrastructures, sans parler des dégâts sur les sols qui menacent la viabilité écologique et l'agriculture.

La couche d'ozone n’est pas épargnée. Les halons et les fréons, substances destructrices de la couche d'ozone sont larguées dans le ciel irakien par les avions de combat dans lesquels ils permettent de réduire les particules d'échappement qui permettraient la captation par les détecteurs ennemis. Bien que le Protocole de Montréal ait permis de réduire l'émission de ces substances, la guerre en Irak à elle seule, correspond à trois mois d'émissions civiles normales à l'échelle mondiale.

Par ailleurs, en plus de la fumée qui recouvrit Bagdad, la combustion des nombreux puits de pétrole provoqua des émissions de particules saturées de pétrole, et additionnées de métaux, qui auront des effets négatifs sur la santé des habitants de toute la région pendant très longtemps. De plus, le déversement de pétrole et d'autres substances toxiques dans le Tigre, l'Euphrate et le Golfe Persique correspond à l'une des pires catastrophes environnementales. 
Les munitions de même que les composantes des artilleries comprennent des PCB ainsi que des nitrates; qu'ils explosent ou pas, ces composés représentent des menaces et pour les écosystèmes et pour la santé publique. Le coût de cette invasion est énorme : à peu près 3000 américains et environ 700000 iraquiens tués (Burnham et al., 2006), pour ne pas parler de tous les autres impliqués (d'autres soldats, des consultants, des journalistes, etc.), une dépense de la part du gouvernement américain dans l'ordre de centaines de milliards de dollars, de graves problèmes moraux et éthiques qui entourent maintenant la région et la politique internationale, un appauvrissement de la population iraquienne (Hassan, 2006) et, finalement, une dégradation visible mais non soulignée par les instances qui détiennent le pouvoir de l'environnement (Public Citizen Global Exchange CorpWatch, 2003).

\section{3) L'invasion au Liban en 2006}

Encore une fois, dans la couverture de la guerre, les médias mettent en lumière les pertes en vies humaines et en infrastructures tout en taisant les impacts environnementaux. Or, ces derniers produisent des effets néfastes qui se font sentir à court, moyen et long termes sur la sécurité, la santé et la vie des populations de toute la région. C'est le cas également pour le Liban victime d'une autre guerre en juillet 2006. En marge du nombre de victimes déclarées (800 Libanais) et des dommages aux infrastructures, le nombre de déplacés, plus de 800.000 Libanais, est aussi alarmant quand on connaît les conditions de fuite suivies des conditions de survie dans les camps de réfugiés. Il est à noter que l'invasion a démantelé en grande partie l'infrastructure du Liban, notons par exemple: l'aéroport, les autoroutes, les bâtiments, les systèmes d'eau et d'hydroélectricité, les communications, etc. et a ravagé le paysage du pays, surtout dans le sud. Comme le souligne encore Jernelov (2006), les dommages aux infrastructures sont étroitement liés à ceux causés à l'environnement.

Le Liban s'atèle de nouveau à la tâche de reconstruction du pays et de son image, tâche qu'il poursuivait depuis la guerre des années quatre-vingts (Jamail, 2006). Cependant, si les infrastructures peuvent être reconstruites avec quelques 20 milliards de dollars évalués (Hersh, 2006) et dans un temps relativement court, le coût des dommages à l'environnement est très difficile à évaluer et leurs effets se feront sentir bien longtemps après la fin de la guerre. Notons quelques exemples.

a) Les plages libanaises, parmi les plus propres de la Méditerranée, sont aujourd'hui souillées; plus de 100.000 tonnes de pétrole ont été déversées (Jernelov, 2006).

b) Les feux de forêt se sont intensifiés sous l'effet de l'explosion des bombes. 
c) Des blessures autres que celles dues aux frappes sont observées : elles seraient la manifestation de brûlures du second degré causées par des produits caustiques alcalins qui se trouvaient dans les bâtiments détruits.

d) Un rapport indique qu'à peu près 40 Libanais par mois meurent actuellement à cause des bombes non explosées (Gendzier, 2006).

e) Les immeubles construits ou restaurés depuis les années quatre-vingts contiennent d'importantes quantités d'amiante utilisée pour l'isolation thermique. Le bombardement de ces immeubles libère des poussières d'amiante qui lorsqu'elles sont inhalées augmente le risque de fibrose pulmonaire et de cancer du poumon. La saga des travailleurs de l'amiante en Occident, aux prises avec l'amiantose, a conduit à l'adoption de rigoureuses mesures de protection au travail. Qu'en est-il pour les victimes des bombardements?

\section{Haïti depuis toujours...}

L'exemple d'Haïti illustre d'une manière frappante le mariage entre le sousdéveloppement et l'environnement. Haïti vit une situation écologique désastreuse et dangereuse pour la sécurité et la santé de la population (Konbit Pou Ayiti, 2006). Le déboisement massif, l'érosion de la terre galopante, l'épuisement et la dégradation des sols, la contamination de l'eau, des maladies de jadis abolies ailleurs, des conditions sanitaires dépassées, la pauvreté qui étouffe la majorité des gens, et une instabilité économique et politique continue, caractérisent la vie en Haïti. Le Ministre haïtien de l'environnement en 2005, monsieur Yves André Wainright, reconnaît que « les indicateurs environnementaux en Haïti dépassent leur seuil d'alerte et la population se trouve dans un état d'extrême vulnérabilité » (Alter Presse, 14 juillet 2005). De plus, chaque catastrophe naturelle, comme par exemple le cyclone Jeanne ayant ravagé la ville des Gonaïves en septembre 2004, accentue encore plus les vulnérabilités du pays et de sa population. Leurs impacts environnementaux doivent être considérés comme des transversaux puisqu'ils affectent tous les secteurs du pays: la santé, l'éducation, l'habitat, les écosystèmes, l'économie, les infrastructures, le social, le politique. Les évaluations effectuées arrivent toutes à la même conclusion : les conséquences catastrophiques de Jeanne sont fortement dues à la vulnérabilité environnementale préexistante. Selon Nancy Roc (2007), Haïti présente déjà toutes les caractéristiques d'une véritable catastrophe écologique : déforestation, érosion accélérée, appauvrissement des sols, perturbations du cycle de l'eau, infrastructures déficientes, sur-population et sur-sollicitation des ressources, relief montagneux et urbanisme anarchique, tous ces facteurs concourent à rendre les populations plus exposées aux colères météorologiques.

Malgré cette situation dramatique, Haïti ne semble pas être considérée comme une zone de crise par les preneurs de décision au niveau international 
(Chomsky et al., 2006). Comment ne pas en être alarmé? Serait-ce parce que Haïti ne détient pas les richesses naturelles qui sont au goût du jour? Serait-ce parce qu'elle n'a pas de poids stratégique sur l'échiquier du monde? Ou encore, seraitce parce que, à l'instar du Rwanda et du Darfour, sa population est noire? Inspirée du géographe Georges Anglade, nous nous demandons comment Haïti sortira du sous-développement pour aller au développement tout en passant par le nécessaire «désenveloppement » de tous les enveloppements néo-coloniaux? (Lévy, 2004).

Les désastres environnementaux mettent l'accent sur l'urgence humanitaire endémique d'Haïti, qui l'empêche d'atteindre un processus de développement durable, de réduction de la pauvreté, de satisfaction aux besoins essentiels et de compétitivité sur le plan commercial. L’inégalité croissante, la pauvreté continue, la décapitalisation des entreprises de l'État ne sont que soulignées par les pluies torrentielles qui ont affecté le pays (CEPALC, 2005, p.62).

\section{5) Les Premières Nations du Canada depuis la « découverte »...}

Les peuples autochtones du Canada, connus et reconnus comme les Premières Nations, vivaient en fonction de la protection et l'harmonie avec l'environnement pendant des milliers d'années jusqu'à l'arrivée des Européens au quinzième siècle (Royal Commission on Aboriginal Peoples, 1991). Les iniquités que l'on observe aujourd'hui témoignent des interactions entre les deux groupes. Les Autochtones luttent depuis toujours d'avoir accès à leurs territoires, négociés dans des ententes qui n'ont pas été toujours respectés par les colonisateurs. Le sous-développement chez les Autochtones est évident en examinant et comparant le standard de vie des deux groupes, la santé, les possibilités d'emploie, le niveau d'emprisonnement, la discrimination et d'autres indicateurs de développement (Mendelson, 2006). Quand les Autochtones revendiquent leurs droits ancestraux à la chasse, à la pêche, et aux territoires (Assembly of First Nations, 2006), la représentation des perspectives des Premières Nations est souvent caractérisée comme une injustice, non envers les Autochtones mais envers le peuple Canadien (Linden, 2006). De plus, l'exploitation effrénée des richesses naturelles (forêts, rivières, lacs, sols, etc.) par le peuple Canadien n'est pas remise en cause, tout au contraire, alors que les territoires autochtones sont constamment réquisitionnés, ce qui fait peser une grande menace sur leur mode de vie et leur tissu social (Boyd, 2003). Protéger l'environnement est souvent perçu comme un ensemble de gestes simples et localisés, comme le recyclage, et non comme un ensemble de facteurs géopolitiques, économiques, sociaux et culturels qui ne s'accordent pas nécessairement avec la notion de progrès axé sur la sur-consommation de biens. Par ailleurs, il est à noter que, sur le territoire nord-américain, la variable raciale a joué et continue encore de jouer un rôle très important dans le statut socio- 
économique effectif des Premières Nations (Canadian Council on Social Development, 2003).

Sommaire de vignettes illustrées

En somme, les dénominateurs communs pour les cinq exemples cités ci-dessus sont les suivants :

- Une convergence des intérêts économiques et politiques pour écarter les groupes marginalisés (McLaren, 2007);

- $\quad$ Un manque d'intérêt flagrant pour l'environnement;

- Une interprétation telle que la justice sociale soit subjuguée à une analyse dominée par les intérêts du (ou des) groupe(s) majoritaire(s) (Freire, 1973);

- Une composante racialisée où les gens de couleur font face à une discrimination systémique importante individuellement et collectivement (Henry \& Tator, 2005);

- Le développement, appuyé par des relations de pouvoir inéquitables incluant la force militaire, est abordé à l'aune de l'hégémonie occidentale.

Nous dénonçons les rapports de pouvoir inéquitables en ce qui a trait à l'environnement. De plus, nous mettons en doute la capacité de l'éducation d'interroger de manière critique les enjeux socio-environnementaux. Dans un temps où le néo-libéralisme semble prendre de l'avant sur l'aspect humain et démocratique, nous encourageons un débat sur la dynamique transversale de l'environnement, vu à la fois comme une dimension sociale, politique, éducationnelle et un outil de résistance.

Une inflation galopante des risques et des vulnérabilités qu’ils engendrent La vulnérabilité se distingue de la pauvreté en ce sens qu'elle constitue l'ensemble des facteurs qui poussent les gens dans la pauvreté, les y maintiennent et en empêchent la sortie (Action Aid, 2005, cité par Yahmin, Rahman et Huq, 2006). Elle est définie comme « les conditions déterminées par des facteurs ou des processus physiques, sociaux, économiques et environnementaux qui accroissent la sensibilité d'une communauté aux effets des risques » (ONU, traité de Hyogo, 2005). Elle est aussi décrite comme une « absence de défense face à l’insécurité et l'exposition aux risques, aux traumatismes et au stress » (Chambers, 1989, cité par Yahmin, Rahman et Huq, 2006). Ces auteurs soulignent l'importance de la notion de vulnérabilité car elle permet de mettre en évidence les dimensions multiples de l'exclusion et les stratégies auxquelles ont recours les victimes pour y faire face. Ils insistent : «Comprendre la vulnérabilité devrait améliorer notre 
interprétation des processus climatiques, sociaux, intergénérationnels, géographiques, économiques et politiques qui génèrent la pauvreté chronique » (p. 119). Les concepts associés sont "faire face ", "adaptation », " résilience » et «empowerment».

La culture...les cultures...

Pour contrer l'invalidation, la marginalisation et l'exclusion des personnes et des savoirs, une rencontre véritable peut avoir lieu. Nous pensons qu'elle n'est possible que si elle sollicite les personnes dans leur identité fondamentale, c'est-àdire, l'individu comme " porteur de culture ", donc de valeurs, de quête et de construction de sens, de vision, de symboles, d'histoire, de questionnements, de croyances, de paroles, d'actes, de savoirs, d'espérance. «La culture, disait Herskovits, est la part de l'environnement fabriquée, construite par l'homme » (cité par Camilleri, 1985, p.8). La culture est ici comprise dans son sens anthropologique, c'est-à-dire, qu'aucune femme, aucun homme n'est « en soi », une sorte de réalité objective indépendante. Femme et homme sont saisis et abordés selon la place qu'elle et lui occupent dans la nature et dans le monde social, ses hiérarchies et ses divisions; ses finalités et les fonctions attribuées; dans leur statut et les rôles qu'ils se voient attribuer, sans oublier les associations conscientes et inconscientes dues aux expériences du quotidien. Ainsi, pour Camilleri :

la culture est l'ensemble plus ou moins lié des significations acquises les plus persistantes et les plus partagées que les membres d'un groupe, de par leur affiliation à ce groupe, sont amenés à distribuer de façon prévalente sur les stimuli provenant de leur environnement et d'euxmêmes, induisant vis-à-vis de ces stimuli des attitudes, des représentations et des comportements communs valorisés, dont ils tendent à assurer la reproduction par des voies non génétiques (1985, p.13).

$\mathrm{Si}$, au singulier, "la culture » anthropologique désigne cet ancrage biologique, physique et social dans lequel toute femme et tout homme est abordé, par contre, au pluriel, «les cultures » renvoient aux diverses modalités de cet ancrage biophysico-social. Nous nous référons à l'anthropologue Edward T. Hall pour comprendre la centralité et la profondeur de cet ancrage. Cette dimension de la culture a trait à la plus fondamentale et la plus indicible des expériences, celle qu'il désigne comme le niveau de culture primaire (inconscient et implicite), par opposition aux niveaux de culture secondaire (conscient et implicite) et tertiaire (conscient et explicite). Le niveau de culture primaire est sous-jacent, structuré, implicite, inconscient, mais déterminant. C'est « une sorte de grammaire culturelle qui détermine la manière dont les individus perçoivent leur 
environnement, définissent leurs valeurs et établissent leur cadence et leurs rythmes fondamentaux » (Hall, 1984, p.14).

\section{L'interculturel en contexte d'acculturation}

L'interculturel a toujours existé. L'humanité ne serait pas ce qu'elle est sans la rencontre des cultures. Cela nous amène à considérer toute culture dans son essence comme le fruit de la rencontre et de l'interpénétration de plusieurs cultures. De tout temps, même en s'affrontant, les humains ont toujours échangé des aliments, des biens, des produits de luxe, des façons de faire, des techniques, des arts, des amours, des philosophies, des questionnements et des raisons d'espérer. D'où vient donc ce nouveau problème avec l'interculturel? Qu'est-ce donc qui pose problème à notre époque?

Les écrits ayant trait à l'interculturel commencent souvent par un tableau des migrations selon un vecteur de déplacement du Sud vers le Nord, et poursuivent avec les modalités d'acculturation des première, deuxième et même troisième générations, dans leurs parcours socio-scolaires et socio-professionnels. L'interculturel semble considéré lorsqu'il s'agit de peuples aux phénotypes différents mis en présence l'un de l'autre à la suite de mouvements migratoires, volontaires ou involontaires, et résultant en la vulnérabilité des migrants et de leurs descendants. L'interculturel est également désigné lorsqu'il s'agit de groupes qui se voient imposer une culture autre sur leur propre territoire, comme c'est le cas pour les Autochtones d'Amérique face aux premiers arrivants d'Europe par exemple. Plus précisément, l'interculturel semble problématique lorsqu'il s'agit du processus d'acculturation où préexistent des rapports inéquitables de pouvoir entre les deux groupes en contact. Le groupe acculturé, dit minoritaire (numériquement, économiquement, politiquement ou autrement), va voir sa culture se transformer radicalement sous la pression de la culture dominante et y fera face en adoptant diverses stratégies d'acculturation (Segall et al., 1999, p.305).

Les facteurs qui affectent les stratégies d'acculturation sont nombreux, notamment: la société d'origine, la société d'accueil ainsi que les facteurs qui préexistent à la rencontre des deux groupes, tels la distance culturelle (Segall et al., 1999, p.316). Nous posons que l'un de ces facteurs préexistant au contact entre les deux groupes est l'histoire des rapports de pouvoir et de savoir entre leurs deux cultures. Autrement dit, étant donné la rémanence et la prégnance du racisme épistémologique dans l'histoire des rapports entre les populations du Sud et du Nord, on peut avancer que ce racisme épistémologique constitue un obstacle majeur, incontournable même, qui perturbe les stratégies d'acculturation et met en péril la rencontre interculturelle. Ce qui permet de postuler la centralité du racisme dans la compréhension des phénomènes interculturels. 
Par ailleurs, le regard interculturel selon le vecteur du Nord vers le Sud se révèle également problématique. Voici quelques exemples d'analogies à déplorer et à dé-construire:

- Le musée ethnographique ou « zoo humain »

- Le tourisme traditionnel ou nouveaux voyages de «Tintin» en Afrique

- L'aide humanitaire ou charité bien ordonnée commence par soi

- La couverture médiatique ou mise en scène de l'Autre sans l'Autre

- La coopération internationale ou démonstration à l'Autre du savoir-faire technologique

- La mondialisation ou néo-colonialisme

- L'invasion culturelle, politique, militaire, économique ou démocratisation

- Le libre-échange commercial et culturel ou mise en tutelle de l'Autre

- La recherche interculturelle ou discours sur l'Autre sans l'Autre

L’interculturel ne peut être que, si et seulement si, il y a :

- Rencontre de l'Autre

- Reconnaissance de L'Autre

- Dialogue avec l'Autre

- Respect mutuel des différences

- Apport réciproque

- Convergence

L'interculturel en environnement

Où sont les cultures en environnement? Où se rencontrent-elles? La rencontre des cultures a-t-elle lieu en éducation relative à l'environnement et, si oui, comment se fait-elle? Poser ces questions, c'est s'attarder au carrefour du lien entre nature et culture et au carrefour des liens entre cultures. Pourtant, la culture, encore moins l'interculturel, n'est pas très explicitée en environnement

En environnement, la notion de culture semble étroitement liée à celle de communautés locales ancrées dans leur espace territorial et dans leur espace temps.

Il y a, en effet, de bonnes raisons de penser que c'est essentiellement à l'échelle des territoires que pourront être construites, démocratiquement, les articulations indispensables entre les dimensions sociales et écologiques du développement durable (Theys, cité par von Frenckell, 2005 p.9). 
C'est au niveau local que la participation active des individus et groupes sociaux à la solution des problèmes environnementaux et les opportunités de donner aux apprenants la possibilité de prendre des décisions peuvent prendre sens et que se construisent des attitudes relatives au « pouvoir agir (von Frenckell, 2005, p.8)

Cette auteure fait d'ailleurs remarquer combien, en réaction au modèle unique du discours environnemental, les singularités territoriales tendent à se renforcer sur la scène institutionnelle dans une logique de décentralisation et de réappropriation : «des représentations individuelles et collectives, sociales et culturelles, des dimensions identitaire, symbolique, politique, historique, etc. " (von Frenckell, 2005, p.9) En ce sens, le territoire devient : «la volonté d’un groupe social de tisser du lien social localisé, de créer des solidarités à l'échelle locale, de se vouloir co-auteur d'un développement de proximité » (Di Meo, 1998, cité par Frenckell, 2005, p.9). Ainsi est née dans les années quatre-vingt-dix la notion de « communauté d'apprentissage », un courant « en quête de valeurs, de principes et de modes de vie alternatifs (...) faire face à la crise environnementale et aux effets pervers des tendances dominantes de développement, associées à un ordre mondial axé sur une croissance sans frein » (Orellana, 2005, p.67). L'aliénation des individus et des groupes sociaux est centrale dans ce processus qui «a amené les communautés humaines à développer des relations qui se sont révélées destructrices pour le milieu de vie, en le dégradant, le contaminant, épuisant ses ressources, réduisant et mettant en péril sa biodiversité et aussi faisant disparaître et méprisant des cultures et savoirs millénaires » (Idem).

Les discours écologiques adroitement récupérés en «développement durable » et en "éducation au développement durable » peuvent sembler salvateurs en mettant l'accent sur la sauvegarde du patrimoine des générations futures. Cependant, comme le recommande Theys, la vigilance est toujours de mise et exige que l'on se demande aussi « si sous couvert d'intentions louables, se référant aux générations futures, une élite technologique, armée d'un discours moralisateur sur l'état de la planète et les responsabilités de chacun, n'est pas en train d'imposer une nouvelle vision du monde, un «nouvel évangile » auquel personne n’aurait explicitement souscrit » (cité par von Frenckell, 2005, p.9-10)

Cette vigilance dont parle Theys est appelée " posture critique " par certains et «posture de résistance » par d'autres. Dans les deux cas, de nouvelles épistémologiques se font jour. Selon Leff (2005), la crise environnementale est la première crise du monde global produite par la méconnaissance de la connaissance. La connaissance ne représente plus la réalité; elle ne sauve plus, ne soigne plus et n’offre plus de sécurité face aux divers risques. L'épistémologie environnementale amène justement à « une politique de la diversité culturelle et de la différence; elle s'ouvre à un dialogue intersubjectif et interculturel au-delà 
de l'espace d'un échange interdisciplinaire ». Quelques principes se dégagent de sa réflexion :

- L'épistémologie environnementale donne cours à un nouveau savoir, conduit non seulement à un changement d'épistémè, mais plutôt à une diversité épistémologique, une diversité de rationalités, une diversité d'imaginaires qui valorisent les valeurs et savoirs qui lient les différentes cultures à la nature

- L'épistémologie environnementale est une politique du savoir qui lie les conditions uniques de vie sur la planète avec l'énigmatique existence et le désir de vie de l'être humain

- L'épistémologie environnementale reconnaît les effets des formes de connaissance dans la construction/destruction de la réalité

- Face à la volonté de résoudre la crise écologique par un "contrôle rationnel de l'environnement », le savoir environnemental questionne l'irrationalité de la raison scientifique de même que les limites de la science normale à appréhender l'environnement

- L'épistémologie environnementale navigue vers de nouveaux horizons de l'Être et du temps, de nouvelles relations entre l'Être et le savoir, cet Être constitué par sa culture dans les différents contextes dans lesquels il attribue un sens à la nature, reconfigure ses identités et forge ses modes de vie.

L'épistémologie environnementale se place selon l'angle de la perspective critique (Adorno; Habermas; Robottom et Hart; Sauvé). Selon Sauvé (2005, p.37), la perspective critique de recherche en éducation relative à l'environnement «se préoccupe de déconstruire les réalités socio-environnementales, en vue de mieux en analyser les composantes (en questionnant les évidences, les idées reçues, les hypothèses, les valeurs sous-jacentes, les rapports de pouvoir) pour enfin reconstruire une réalité jugée plus appropriée en ce qui concerne le réseau des relations personne - société - environnement ».

On peut dire que la posture critique est le prêt-à-porter qui seyait le mieux jusqu'à présent aux intellectuels des groupes marginalisés. En effet, les premiers travaux sur les questions du racisme et sur la théorie anti-raciste ont été élaborés dans ce cadre alors même que les milieux académiques y étaient sévèrement hostiles. Cependant, la tradition critique a émergé elle aussi des histoires sociales européennes et ne permet pas d'avancées plus importantes dans le développement d'une conscience critique et d'attitudes appropriées pour les personnes et les groupes dominés. La tradition critique fut donc nécessaire à l'émergence d'une approche favorable aux chercheurs issus des groupes marginalisés mais se révèle insuffisante à sa consolidation. 
Adoptant une posture de résistance, d'autres chercheurs (Carr; Dei; Nandy; Saïd; Shiva; Smith; Thésée) proposent une épistémologie de la décolonisation appelant à : la dé-colonisation des ontologies (principes fondamentaux), la dé-colonisation des épistémologies (savoirs), la dé-colonisation des axiologies (valeurs) et la dé-colonisation des méthodologies (procédures). Pour ce, une rupture d'avec les épistémologies courantes est nécessaire. Même le paradigme critique ne suffit plus. Le champ de l'environnement au sens de «Oïkos », à la fois comme domaine de recherche et d'éducation, peut être vu comme un site significatif de combat entre l'épistémologie hégémonique et les épistémologies alter-natives (nées autrement). En fait, l'interculturel ou rencontre véritable des cultures ne peut avoir lieu sans cette posture de résistance qui requiert des groupes marginalisés de se ré-approprier leur Être confisqué : 1) en refusant les savoirs toxiques pour l'identité; 2) en re-questionnant les savoirs appris; 3) en re-construisant les savoirs nécessaires; 4) en se ré-appropriant l'espace mental et l'espace social, par la parole, la mémoire, la participation active et la prise de pouvoir sur soi (Thésée, 2006; Solar, 1988).

À l'instar des signataires de la Déclaration de Durban qui s'engage à appuyer la construction d'un mouvement mondial de base pour une justice climatique, nous proposons une posture socio-épistémologique de résistance pour dé-coloniser l'édifice des savoirs et contrer les discours qui néo-colonisent la pensée tout en renforçant encore et toujours les injustices sociale et environnementale. Réclamons la justice sociale, maintenant! Réclamons la justice environnementale, maintenant!

\section{Conclusion}

En constatant que, à court et moyen termes, tous ne partagent pas le même destin climatique, nous avons mené une réflexion qui articule les éléments qui nous semblaient importants : d'un côté l'environnement, les menaces climatiques, les vulnérabilités multiples et l'injustice environnementale, et de l'autre, le colonialisme, le racisme épistémologique et l'injustice sociale. En les mettant face à face, nous avons tenté de démontrer le chevauchement des injustices sociales et environnementales. Optimistes, nous avons voulu voir dans l'interculturel une possibilité de rencontre des cultures, rencontre devenue incontournable dans la nouvelle perspective environnementale. Cependant, pour qu'une véritable rencontre des cultures ait effectivement lieu, les cultures marginalisées doivent adopter une posture de résistance, une sorte de colonne vertébrale épistémologique, qui leur permettra d'être d'abord soi pour pouvoir aller à la rencontre de l'Autre. 


\section{Bibliographie}

Alter Presse. 14 juillet 2005. Consulté le 3 février 2007 sur http://www.alterpresse.org/spip.php?article2512 .

Assembly of First Nations. (2006). Environmental Sterwardship. Consulté le 2 novembre 2006 sur http://www.afn.ca/article.asp?id=23 .

Boyd, D. (2003). Unnatural Law - Rethinking Canadian Environmental Law andPolicy. Vancouver: UBC Press.

Burnham, G. et al. (2006). Mortality after the 2003 invasion of Iraq: a cross-sectional cluster sample survey. Consulté le 3 novembre 2006 sur http://www.westpointgradsagainstthewar.org/thelancetstudy.htm .

Camilleri, Carmel. (1985). Anthropologie culturelle et éducation. Lausanne : UNESCO/ Delachaux-Niestlé.

Canadian Council on Social Development. (2003). Aboriginal children in poverty in urban communities: Social exclusion and the growing racialization of poverty in Canada. Consulté le 2 novembre 2006 sur http://www.ccsd.ca/pr/2003/aboriginal.htm .

Carr, P. (2007). The Whiteness of Educational Policymaking. In Carr, P. and Lund, D. (dir.) The Great White North? Exploring Whiteness, Privilege and Identity in Education. Rotterdam: Sense Publishers.

Carson, Rachel. (1962). Printemps silencieux. Paris : Éditions Plon.

CEPALC. Commission économique pour l'Amérique Latine et les Caraïbes (2005). Le cyclone Jeanne en Haïti : Dégâts et effets sur les départements du Nord-Ouest et de l'Artibonite: Approfondissement de la vulnérabilité. Consulté le 4 novembre 2006 sur http://www.eclac.org/publicaciones/xml/1/20971/L648-1.pdf .

Chomsky, N., Farmer, P., Goodman, A., et Aristide, J-B. (2006). Getting Haiti Right This Time: The U.S. and the Coup. Monroe, Maine: Common Courage Press.

Déclaration de Durban sur le commerce du carbone. (2006). "La justice climatique, maintenant!" In Changements climatiques. Impasses et perspectives. Points de vue du Sud., (p. 199-202). Louvain-la-Neuve : Centre Tricontinental et Éditions Syllepse.

Dei, G., Karumanchery, L. et Karumanchery-Luik, N. (2004). Playing the Race Card: Exposing White Power and Privilege. New York: Peter Lang.

Fanon, Frantz. (1967). A Dying Colonialism. New York: Grove Press.

Foucault, Michel. (1969). L'archéologie du savoir. Paris : Gallimard, coll. « Bibliothèque des Sciences humaines »

Freire, P. (2005). Pedagogy of the oppressed. New York : Continuum.

Gendzier, I. (2006). Exporting death as democracy: an essay on U.S. foreign policy in Lebanon, ZNet. Consulté le 2 novembre 2006 sur http://www.zmag.org/content/showarticle.cfm?ItemID=11123 .

Hall, Edward T. (1984). La danse de la vie. Temps culturel. Temps vécu. Paris: Seuil.

Hassan, G. (2006). The Case For Iraqi Genocide, Countercurrents.org. Consulté le 2 novembre 2006 sur http://www.countercurrents.org/iraq-hassan271006.htm .

Henry, F. et Tator, C. (2005). The colour of democracy: racism in Canadian society. Toronto: Nelson Thompson.

Herrnstein, Richard \& Murray, Charles. (1994). The Bell Curve: Intelligence and class structure in American life. New York : Free Press. 
Hersh, S. (2006). Watching Lebanon: Washington's interests in Israel's war. The New Yorker, August 21. Consulté le 4 novembre 2006sur http://www.newyorker.com/fact/content/articles/060821fa_fact .

Jamail, D. (2006). Tomgram: Dahr Jamail, The Damage in Lebanon -- and Beyond. Consulté le 3 novembre 2006 sur http://www.tomdispatch.com/index.mhtml?pid=109209 .

Jerneloy, Arne. (2006). La guerre du Liban et l'environnement. Consulté le 5 janvier 2007 sur http://www.project-syndicate.org/commentary/jernelov8/French .

Jerneloy, Arne. (2003). La guerre et l'environnement. Consulté le 5 janvier 2007 sur http://www.project-syndicate.org/commentary/jernelov2

Jerneloy, Arne. (2003). Les conséquences sur l'environnement de la guerre en Irak. Consulté le 6 janvier 2007 sur http://www.project-syndicate.org/commentary/jernelov1/French .

Jinhua, Dai. (2006). Chine : les nouvelles voies de la résistance. In Changements climatiques. Impasses et perspectives. Points de vue du Sud (p. 201 -205). Louvain-la-Neuve : Centre Tricontinental et Éditions Syllepse.

Khor, Martin. (2006). Conséquences de certaines règles de l’OMC sur la poursuite des OMD. In Alternatives Sud (dir.), Objectifs du millénaire pour le développement. Points de vue critiques du Sud. (p.67-99). Louvain-la-Neuve: Centre Tricontinental et Éditions Syllepse.

Konbit Pou Ayiti (2006). Haiti's environment. Consulté le 28 octobre 2006 sur http://www.konpay.org/wordpress/environment/ .

Leff Zimmerman, Enrique. (2006). Géopolitique de la diversité et développement durable. In Changements climatiques. Impasses et perspectives. Points de vue du Sud (p.185-196). Louvain-la-Neuve : Centre Tricontinental et Éditions Syllepse.

Leff Zimmerman, Enrique. (2005). Savoir environnemental : épistémologie, rationalité et dialogue de savoirs. Enjeux et défis pour l'éducation. In Sauvé, L., Orellana, I. et van Steenberghe, É. (dir.), Éducation et environnement. Un croisement de savoirs (p.49-65). Montréal : ACFAS.

Lévy, Joseph J. (2004). Entretiens avec Georges Anglade. L'espace d'une génération. Montréal : Liber.

Lewis, C. L. R. (2001). Black Jacobins: Toussaint L'Ouverture and the San Domingo revolution. London: Penguin.

Linden, S. (2006). The Ipperwash Inquiry. Consulté le 28 octobre 2006 sur http://www.ipperwashinquiry.ca/ .

McLaren, P. (2007). Life in schools: An introduction to critical pedagogy in the foundations of education. New York: Longman.

Media Awareness Network. (2007). Teaching backgrounder: Hurricane Katrina. Consulté le 28 mars 2007 sur http://www.media- awareness.ca/english/ resources/educational/teachable_moments/hurricane_katrina.cfm .

Mendelson, M. (2006). Aboriginal peoples and postsecondary education in Canada. Ottawa: The Caledon Institute of Social Policy.

Orellana, Isabel. (2005). L’émergence de la communauté d’apprentissage. In Sauvé, L., Orellana, I. et van Steenberghe, É. (dir.), Éducation et environnement. Un croisement de savoirs (p.67-83). Montréal : ACFAS.

Pardo, Thierry. (2002). Éléments d'ethnopédagogie pour l'éducation relative à l'environnement. La Caunette : Éditions de Babio. 
Payne, K. (2005). The media as an instrument of war, Parameters, Spring, 81-93.

Public Citizen Global Exchange CorpWatch (2003). Bechtel: Profiting from destruction: Why the corporate invasion of Iraq must stop. Consulté le 3 janvier 2007 sur http://www.citizen.org/documents/profilebechtel.pdf .

Rickford, John. (2005). Racism in media language and law enforcement officers' actions after Hurrican Katrina. Consulté le 24 mars 2007 sur http://72.14.205.104/search?q=cache:uhhXUpzSK_sJ:ccsre.stanford.edu/pdfs/ JRR_KatrinaRemarks05.pdf+Rickford+2005+racism\&hl=en\&ct=clnk\&cd=1

Roc, Nancy. (2007). Réfugiés environnementaux: un nouveau défi planétaire. Consulté le 6 février 2007 sur http://www.google.ca/search?hl=fr\&q=Cara\%C3\%AFbes+Panos+Institute+Na ncy+Roc\&btnG=Recherche+Google\&meta $=$.

Royal Commission on Aboriginal Peoples (1991). Report of the Royal Commission on Aboriginal peoples. Ottawa. Department of Indian and Northern Affairs, Canada.

Said, Edward. (1979). Orientalism. New York: Vintage Books Edition.

Sauvé, Lucie. (1996) Environmental Education and Sustainable Development: Further Appraisal, Canadian Journal of Environmental Education, 1, p.7-34.

Sauvé, L. et Godmaire, H. (2005).Une problématique d'éducation à la santé environnementale au lac Saint-Pierre. Exploration de la dimension socioculturelle. Éducation relative à l'environnement. Regards. Recherches. Réflexion, 5, p. 15-32.

Sauvé, Lucie. (2005). Repères pour la recherche en éducation relative à l'environnement. In Sauvé, L., Orellana, I. et van Steenberghe, É. (dir.), Éducation et environnement. Un croisement de savoirs (p.27-47). Montréal : ACFAS.

Scheurich, James et Young, Michelle. (2002). Coloring epistemology : Are our research epistemologies racially based? In James Scheurich (dir.), Anti-racist scholarship. An Advocacy (p.51-73). New York: State University of New York Press.

Segall, Marshall, Dasen, Pierre, Berry, John et Ype, H. Poortinga. (1999). Human behavior in global perspective. An introduction to cross-cultural psychology. Boston : Allyn and Bacon.

Sharma, Anju. (2006). Énergie, développement, climat et pauvreté. In Changements climatiques. Impasses et perspectives. Points de vue critiques du Sud (p.139152). Louvain-la-Neuve : Centre Tricontinental et Éditions Syllepse.

Shiva, Vandana. (1998). Western science and its destruction of local knowledge. In M. Rahmena et V. Bawtree (dir.). The Post-Development Reader. Halifax : Fernwood Publishing.

Solar, Claudie. (1988). Peindre la pédagogie sur une toile d’équité. In Claudie Solar (dir.), Pédagogie et équité (p.25-65). Montréal: Les Éditions Logique.

Thésée, Gina. (2006). A tool of massive erosion: Scientific knowledge in the neo-colonial enterprise. In George J. Sefa Dei and Arlo Kempf (dir.), Anti-Colonialism and Education: the Politics of Resistance.. Rotterdam: Sense Publishers.

Thuillier, Pierre. (1988). D’Archimède à Einstein: Les faces cachées de l'invention scientifique. Paris: Fayard.

von Franckell, Marianne. (2005). Contextualisation des pratiques et des recherches en éducation relative à l'environnement - Ancrage territorial et culturel. Éducation relative à l'environnement. Regards. Recherches. Réflexion, 5, p.7-11.

Weinstein, B. et Segal, A. (1992). Haiti: The Failure of Politics. Westport, CT: Praeger Publishers. 
Willinsky, John. (1998). Learning to Divide the World. Education at Empire's End. Minneapolis: University of Minnesota Press.

Yamin, F., Rahman, A. et Huq, S. (2006). Vulnérabilité, adaptation et catastrophes climatiques. Dans Changements climatiques. Impasses et perspectives. Points de vue du Sud (p.113-137). Louvain-la-Neuve : Centre Tricontinental et Éditions Syllepse.

Yamgnane, Koffi. (1999). Préface. Dans Savoirs du Sud. Réseau Réciprocité des Relations Nord-Sud (p.7-8). Paris : Éditions Charles-Léopold Mayer.

Paul R. Carr is Assistant Professor at Youngstown State University, where he teaches courses in the sociology of education, and undertakes research on democracy and social justice. He co-edited a book entitled The Great White North? Exploring Whiteness, Privilege and Identity in Education (Sense Publishers). He can be contacted at prcarr@ysu.edu .

Gina Thésée is Assistant Professor at the Université du Québec à Montréal, where she teaches science education and pedagogy. Her research focuses on the epistemology of education and intercultural education. She is presently editing a book entitled Les faces cachées de la recherche interculturelle, which will be published by L'Harmattan in late 2008. She can be contacted at thesee.gina@uqam.ca 